\title{
Contents
}

Part I. Physical, Mathematical, and Numerical Principles

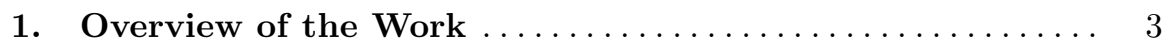

1.1 Part I: Theory ............................. 3

1.2 Part II: Applications ............................ 9

1.3 Part III: Program System $\ldots . \ldots \ldots \ldots \ldots \ldots \ldots \ldots \ldots . \ldots \ldots$

2. Historical Background $\ldots \ldots \ldots \ldots \ldots \ldots \ldots \ldots \ldots \ldots \ldots$

2.1 Milestones in the History of Celestial Mechanics of the Planetary System ....................... 19

2.2 The Advent of Space Geodesy .................... 31

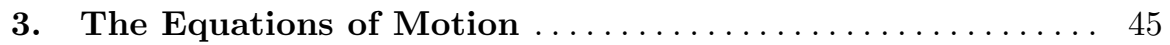

3.1 Basic Concepts............................ 46

3.2 The Planetary System ....................... 50

3.2.1 Equations of Motion of the Planetary System ....... 51

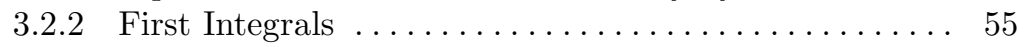

3.3 The Earth-Moon-Sun-System $\ldots \ldots \ldots \ldots \ldots \ldots \ldots \ldots \ldots$

3.3.1 Introduction .......................... 61

3.3.2 Kinematics of Rigid Bodies ................ 63

3.3.3 The Equations of Motion in the Inertial System ..... 71

3.3.4 The Equations of Motion in the Body-Fixed Systems . 78

3.3.5 Development of the Equations of Motion .......... 80

3.3.6 Second Order Differential Equations for the Euler Angles $\Psi, \varepsilon$ and $\Theta \ldots \ldots \ldots \ldots \ldots . \ldots 9$

3.3.7 Kinematics of the Non-Rigid Earth . . . . . . . . . . . 91

3.3.8 Liouville-Euler Equations of Earth Rotation ........ 94

3.4 Equations of Motion for an Artificial Earth Satellite ....... 96

3.4.1 Introduction ....................... 96

3.4.2 Equations for the Center of Mass of a Satellite ...... 97

3.4.3 Attitude of a Satellite ....................... 110

3.5 Relativistic Versions of the Equations of Motion .......... 116

3.6 The Equations of Motion in Overview ................ 120 
4. The Two- and the Three-Body Problems ............ 123

4.1 The Two-Body Problem .......................... 123

4.1.1 Orbital Plane and Law of Areas ................ 123

4.1.2 Shape and Size of the Orbit ................ 125

4.1.3 The Laplace Integral and the Laplace Vector $\boldsymbol{q}$. . . . . 130

4.1.4 True Anomaly $v$ as a Function of Time:

Conventional Approach ....................... 132

4.1.5 True Anomaly $v$ as a Function of Time:

Alternative Approaches ...................... 137

4.2 State Vector and Orbital Elements . . . . . . . . . . . . . . . . 140

4.2.1 State Vector $\rightarrow$ Orbital Elements ................ 142

4.2.2 Orbital elements $\rightarrow$ State Vector . . . . . . . . . . . . . 143

4.3 Osculating and Mean Elements......................... 144

4.4 The Relativistic Two-Body Problem . . . . . . . . . . . . 147

4.5 The Three-Body Problem $\ldots \ldots \ldots \ldots \ldots \ldots \ldots \ldots \ldots \ldots$

4.5.1 The General Problem ......................... 152

4.5.2 The Problème Restreint ..................... 155

5. Variational Equations ......................... 175

5.1 Motivation and Overview ........................ 175

5.2 Primary and Variational Equations ............... 176

5.3 Variational Equations of the Two-Body Problem ......... 183

$5.3 .1 \quad$ Elliptic Orbits . . . . . . . . . . . . . . . . . . . 186

5.3 .2 Parabolic Orbits . . . . . . . . . . . . . . . . . . . 190

5.3 .3 Hyperbolic Orbits . . . . . . . . . . . . . . . . . . . 192

5.3.4 Summary and Examples.................... 193

5.4 Variational Equations Associated with One Trajectory ..... 195

5.5 Variational Equations Associated with the $N$-Body Problem . 198

5.6 Efficient Solution of the Variational Equations .......... 202

5.6.1 Trajectories of Individual Bodies . . . . . . . . . . . 203

5.6 .2 The $N$-Body Problem ....................... 205

5.7 Variational Equations and Error Propagation ........... 206

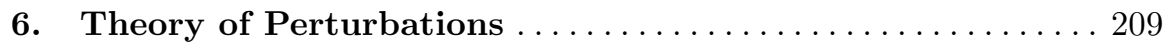

6.1 Motivation and Classification ..................... 209

6.2 Encke-Type Equations of Motion . . . . . . . . . . . . 211

6.3 Gaussian Perturbation Equations .................. 215

6.3.1 General Form of the Equations .................. 215

6.3.2 The Equation for the Semi-major Axis $a \ldots \ldots \ldots 217$

6.3.3 The Gaussian Equations in Terms of Vectors $\boldsymbol{h}, \boldsymbol{q} \ldots 218$

6.3.4 Gaussian Perturbation Equations in Standard Form . . 223

6.3.5 Decompositions of the Perturbation Term .......... 228

6.4 Lagrange's Planetary Equations . . . . . . . . . . . . . 232

6.4.1 General Form of the Equations ................. 232

6.4.2 Lagrange's Equation for the Semi-major Axis $a \ldots \ldots 234$ 
6.4.3 Lagrange's Planetary Equations . . . . . . . . . . . . . . . . 234

6.5 First- and Higher-Order Perturbations . . . . . . . . . . . . . . . 240

6.6 Development of the Perturbation Function . . . . . . . . . . . . . 242

6.6.1 General Perturbation Theory

Applied to Planetary Motion ................ 243

6.7 Perturbation Equation for the Mean Anomaly $\sigma(t) \ldots . . .247$

\section{Numerical Solution of Ordinary Differential}

Equations: Principles and Concepts . . . . . . . . . . 253

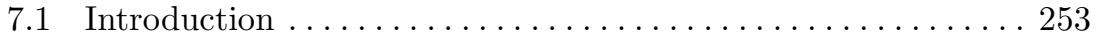

7.2 Mathematical Structure . . . . . . . . . . . . . . . . . . 255

7.3 Euler's Algorithm . . . . . . . . . . . . . . . . . . . . 259

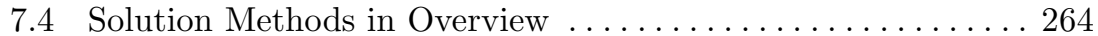

7.4.1 Collocation Methods ........................ 264

7.4 .2 Multistep Methods .................... 266

7.4 .3 Taylor Series Methods ...................... 269

7.4 .4 Runge-Kutta Methods . . . . . . . . . . . . . . . 271

7.4 .5 Extrapolation Methods................... 275

7.4.6 Comparison of Different Methods.............. 277

7.5 Collocation . . . . . . . . . . . . . . . . . . . . . . . . 279

7.5.1 Solution of the Initial Value Problem . . . . . . . . . 280

7.5.2 The Local Boundary Value Problem . . . . . . . . . . . . 283

7.5.3 Efficient Solution of the Initial Value Problem... . . . . . 285

7.5.4 Integrating a Two-Body Orbit with a High-Order Collocation Method: An Example. . 291

7.5.5 Local Error Control with Collocation Algorithms . . . . . 295

7.5.6 Multistep Methods as Special Collocation Methods . . . 304

7.6 Linear Differential Equation Systems

and Numerical Quadrature . . . . . . . . . . . . . . . 312

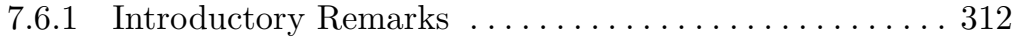

7.6 .2 Taylor Series Solution . . . . . . . . . . . . . . . . . . 313

7.6.3 Collocation for Linear Systems: Basics . . . . . . . . . . . 315

7.6.4 Collocation: Structure of the Local Error Function . . . . 317

7.6.5 Collocation Applied to Numerical Quadrature . . . . . . . 320

7.6.6 Collocation: Examples ................... 324

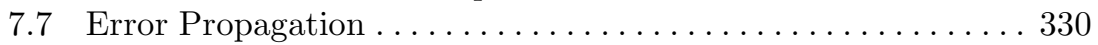

7.7.1 Rounding Errors in Digital Computers . . . . . . . . . . . 332

7.7.2 Propagation of Rounding Errors . . . . . . . . . . . 334

7.7.3 Propagation of Approximation Errors . . . . . . . . . . . 341

7.7.4 A Rule of Thumb for Integrating Orbits of Small Eccentricities with Constant Stepsize Methods . . . . . . . . . . . 348

7.7.5 The General Law of Error Propagation . . . . . . . . . . . 350 
8. Orbit Determination and Parameter Estimation ......... 355

8.1 Orbit Determination as a Parameter Estimation Problem ... 355

8.2 The Classical Pure Orbit Determination Problem ......... 356

8.2.1 Solution of the Classical Orbit Improvement Problem . 357

8.2 .2 Astrometric Positions ....................... 363

8.3 First Orbit Determination ..................... 366

8.3.1 Determination of a Circular Orbit ............ 369

8.3.2 The Two-Body Problem as a Boundary Value Problem 373

8.3.3 Orbit Determination as a Boundary Value Problem . . 378

8.3 .4 Examples .......................... 381

8.3.5 Determination of a Parabolic Orbit ............ 384

8.3.6 Gaussian- vs. Laplacian-Type Orbit Determination ... 388

8.4 Orbit Improvement: Examples . . . . . . . . . . . . . . . . 396

8.5 Parameter Estimation in Satellite Geodesy............... . 404

8.5.1 The General Task ........................ 405

8.5.2 Satellite Laser Ranging . . . . . . . . . . . . . . . . . 406

8.5.3 Scientific Use of the GPS . . . . . . . . . . . . . . 413

8.5.4 Orbit Determination for Low Earth Orbiters ........ 423

References ................................... 441

Abbreviations and Acronyms ........................ 449

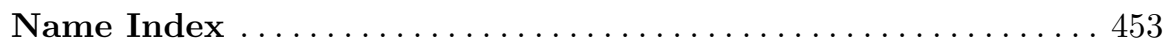

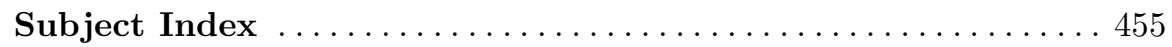

